\title{
Dynamique de bulles en écoulement cisaillé de type Couette cylindrique en gravité normale et en microgravité
}

\author{
Yann Juaneda et Catherine Colin ${ }^{\mathrm{a}}$ \\ IMFT (UMR 5502 CNRS - INP/ENSEEIHT - UPS), Allée du Professeur Camille Soula, 31400 Toulouse, France
}

Reçu le 31 octobre 2003, accepté le 18 décembre 2003

\begin{abstract}
Résumé - Dans le cadre d'une expérience sur la migration de bulles dans un écoulement de Couette cylindrique, un bilan dynamique sur ces bulles a été formulé. Deux cas ont été traités. Le premier pour des écoulements en condition de gravité terrestre, à nombre de Reynolds de bulle important. Le second dans des conditions de microgravité avec des nombres de Reynolds de bulle inférieurs à l'unité. Le premier régime est fortement inertiel et permet la détermination des coefficients de portance et de traînée s'appliquant dans le bilan dynamique. Le second régime est essentiellement visqueux. Utilisant les développements récents établis dans ce domaine, le bilan de forces prend en compte les effets de paroi et de courbure qui peuvent intervenir. En effet, outre la correction de Fàxen, les effets inertiels et de déformation, de même ordre, sont conjointement pris en compte dans la migration des inclusions. Les particules se trouvant dans ce cas dans une zone intermédiaire entre la très proche paroi et l'écoulement sans frontière, les coefficients des forces inertielles de migration ont été approchés à partir des résultats existants pour des sphères solides.
\end{abstract}

Mots clés : Migration / interaction paroi-bulle / gravité / inertie / déformation

\begin{abstract}
Dynamics of bubbles in a sheared cylindrical Couette flow under normal and micro-gravity conditions. In order to study the migration of bubbles in a cylindrical Couette flow setup, the balance of the forces acting on these particles is established. Two cases are investigated. On the one hand, in normal gravity conditions, bubble Reynolds numbers are large. On the other hand, in microgravity conditions, bubble Reynolds numbers are lower than unity. The first regime is strongly inertial and under these conditions it will be possible to determine lift and drag coefficients. The second one is essentially viscous. Using recent developments in this field, the force balance takes into account wall and curvature effects. Indeed, besides Fàxen's correction, inertial and deformation effects, of same order, are both taken into account. Because particles are found to be in a intermediate area between the near-wall and the unbounded regions, the inertial coefficients are approached from existing results for solid spheres.
\end{abstract}

Key words: Migration / bubble-wall interaction / gravity / inertia / deformation

\section{Introduction}

Les écoulements diphasiques sont présents dans de nombreuses situations pratiques telles que l'ébullition, la cavitation, les réactions chimiques entre le gaz et le liquide dans des colonnes à bulles, la croissance cristalline pour mentionner seulement quelques exemples. Dans la plupart des écoulements en gravité normale, la pesanteur joue un rôle dominant comme force motrice, et pour contrôler la forme des bulles. En revanche, en micropesanteur, les écoulements à bulles sont dominés par d'autres forces : visqueuses, capillaires ou de pression. Les études sur les écoulements diphasiques en micropesanteur sont

\footnotetext{
a Auteur correspondant : colin@imft.fr
}

pilotées par le besoin d'améliorer la compréhension et la prédiction de la dynamique des écoulements diphasiques gaz-liquide. En particulier, afin d'optimiser la conception et le fonctionnement des systèmes thermohydrauliques spatiaux tels que les échangeurs pour le refroidissement de composants électroniques ou d'antennes de satellites ou les séparateurs diphasiques pour le traitement des eaux usées.

Cependant les nombreuses investigations laissent quelques phénomènes de base non expliqués, ces phénomènes se produisant à une échelle intermédiaire de l'écoulement, celle des bulles. L'objet de la présente étude est donc de se focaliser sur l'un de ces phénomènes physiques : la migration des bulles dans un écoulement. 
Tableau 1. Notations et définitions.

\begin{tabular}{|ll|}
\hline Paramètres physiques & \\
$\rho_{\mathrm{f}}\left[\mathrm{kg} \cdot \mathrm{m}^{-3}\right]$ masse volumique de l'huile & $\mu_{\mathrm{f}}[\mathrm{Pa} \cdot \mathrm{s}]$ viscosité dynamique de l'huile \\
$\mu[\mathrm{Pa} . \mathrm{s}]$ viscosité dynamique du gaz de la bulle & $\sigma\left[\mathrm{N} \cdot \mathrm{m}^{-1}\right]$ tension superficielle \\
$R[\mathrm{~m}]$ rayon de la bulle & $r[\mathrm{~m}]$ position radiale du centre de la bulle \\
$l[\mathrm{~m}]$ distance à la paroi & $G$ cisaillement local $\left(\mathrm{s}^{-1}\right)$ \\
$V_{\mathrm{C}}$ vitesse caractéristique du glissement & $V_{\mathrm{S}}^{i}=U_{\mathrm{B}}^{i}-U_{\mathrm{L}}^{i}$ vitesse de glissement $(i=\theta$ ou $r)$ \\
$\lambda=\mu / \mu_{\mathrm{f}}$ rapport des viscosités dynamiques & \\
& \\
Nombres sans dimension & \\
$R_{\mu=}(2+3 \lambda) /(2+2 \lambda)$ & $\alpha$ cisaillement adimensionnel \\
$\kappa=R / l$ distance adimensionnelle & $R e=2 R V_{\mathrm{C}} / \nu$ Reynolds de bulle \\
$\delta=\mu_{\mathrm{f}} V_{\mathrm{C}} / \sigma$ nombre capillaire & $L_{\mathrm{S}}=R / R e=\nu_{\mathrm{f}} / V_{\mathrm{C}}$ longueur de Stokes \\
$L_{\mathrm{G}}=(\nu / G)^{1 / 2}$ Longueur de Saffman & $O h=R \sigma / \rho_{\mathrm{f}} \nu_{\mathrm{f}}^{2}$ nombre d'Ohnesorge \\
$l^{*}=l / L_{\mathrm{G}}$ & $\varepsilon=(G \nu)^{1 / 2} / V_{\mathrm{C}}$ \\
$V_{\mathrm{S}}^{\theta+}=V_{\mathrm{S}}^{\theta} / V_{\mathrm{C}}$ et $V_{\mathrm{S}}^{r+}=V_{\mathrm{S}}^{r} / V_{\mathrm{C}}$ vitesses de glissement & \\
adimensionnelles dans les directions $\theta$ et $r$ & \\
\hline
\end{tabular}

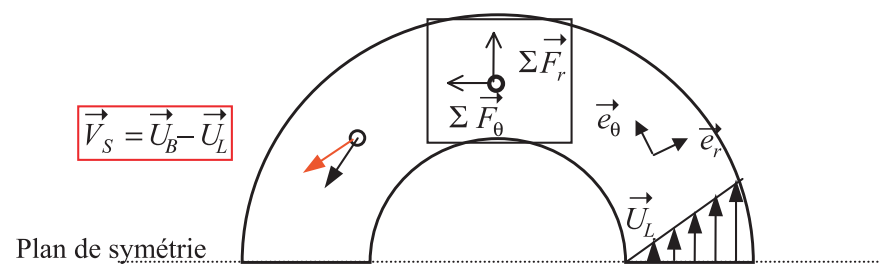

Fig. 1. Schéma du problème.

Cette étude reposera sur des expériences en gravité normale au laboratoire et en conditions de micropesanteur en vols paraboliques. Un dispositif expérimental a été spécialement construit à cette occasion et permet de créer un écoulement cisaillé de type Couette. On cherchera particulièrement à analyser le rôle des forces de pression, viscosité et tension superficielle agissant à l'interface de la bulle.

L'objectif de ce papier est d'écrire des bilans dynamiques cohérents pour des bulles placées dans cet écoulement sous des conditions particulières. Les différents cas traités sont :

- Des bulles de gaz dans du liquide dans des conditions de gravité terrestre.

- Des bulles de gaz dans du liquide en condition de microgravité.

Ces deux cas présentent des particularités propres qui peuvent drastiquement changer les bilans hydrodynamiques. Ainsi, dans le premier cas les nombres de Reynolds de bulle de rayon $R, R e=2 R|\vec{U}-\vec{V}| / \nu_{\mathrm{f}}$, seront importants, $R e \gg 1$ alors que dans le second, les régimes de bulles seront faiblement inertiels : $R e<1$, mais $R e \neq 0$. En conséquence, les bilans écrits pour ces deux conditions seront différents, les phénomènes physiques prépondérants n'étant pas de même nature (Fig. 1).

Les différentes notations utilisées dans ce papier sont rapportées dans le tableau 1.

\section{Dispositif expérimental}

L'étude entreprise tend à caractériser l'influence du cisaillement sur la dynamique des bulles mais aussi sur leur croissance en paroi, leur détachement, leur coalescence, leur déformation et leur éventuelle rupture, le tout en l'absence des forces d'Archimède. Elle est à caractère expérimental, et il a donc été nécessaire de dimensionner un dispositif permettant de réaliser les expériences appropriées. Afin de créer le cisaillement adéquat et de «piéger» les bulles, c'est un écoulement de type Couette entre deux cylindres contrarotatifs qui a été choisi. Il est solution des équations de Navier-Stokes pour le cas de cylindres de rayon $r_{1}$ et $r_{2}\left(r_{2}>r_{1}\right)$ ayant respectivement des vitesses de rotation $\Omega_{1}$ et $\Omega_{2}$ et d'extensions infinies (Guyon et al. [1]) :

$$
\begin{gathered}
\vec{U}(r)=(A r+B / r) \vec{e}_{\theta} \\
\vec{\Omega}=2 A \vec{e}_{z} \\
A=\frac{\Omega_{2} r_{2}^{2}-\Omega_{1} r_{1}^{2}}{r_{2}^{2}-r_{1}^{2}} \text { et } B=\frac{\left(\Omega_{1}-\Omega_{2}\right) r_{1}^{2} r_{2}^{2}}{r_{2}^{2}-r_{1}^{2}}
\end{gathered}
$$

Le terme non nul du tenseur des taux de déformation, $\frac{\partial V_{i}}{\partial x_{j}}$ est un terme non diagonal dans ce cas :

$$
S_{r \theta}=S_{\theta r}=\frac{1}{2}\left(r \frac{\partial}{\partial r}\left(\frac{U_{\theta}}{r}\right)+\frac{1}{r} \frac{\partial U_{r}}{\partial \theta}\right)=\frac{-B}{r^{2}}
$$

Cet écoulement a la particularité de présenter un gradient de cisaillement perpendiculairement aux lignes de courant (Fig. 2).

Pour des raisons de visualisation en présence de g-jitter en microgravité, le dispositif est placé horizontalement.

La dimension de l'entrefer $d=r_{2}-r_{1}$ est nettement plus importante que dans les dispositifs équivalents réalisés jusqu'à présent. En effet en l'absence de la force de flottaison entre les deux phases, le bilan dynamique des bulles au détachement est dominé par les effets de 


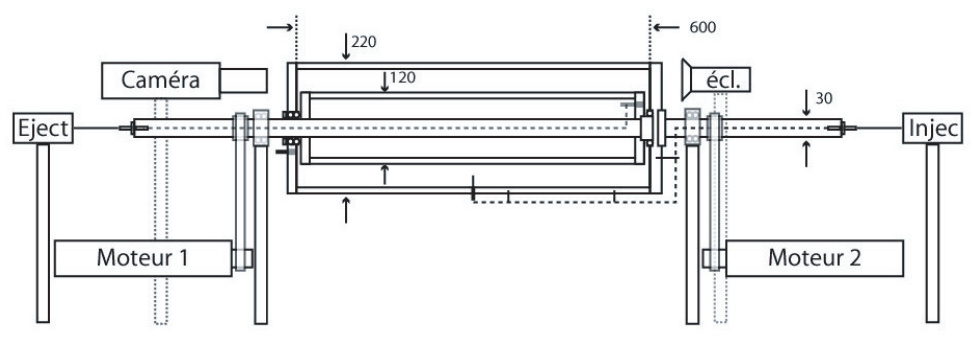

Fig. 2. Schéma du dispositif expérimental.

traînée et de tension superficielle. De fait, les diamètres des bulles formées seront plus importants que dans les écoulements où la force de flottabilité est présente. Les cylindres sont d'une longueur de $600 \mathrm{~mm}$ alors que leur rayons sont respectivement $r_{1}=60 \mathrm{~mm}$ et $r_{2}=100 \mathrm{~mm}$. Les bulles sont injectées au milieu du cylindre extérieur au moyen d'un capillaire amovible. La phase fluide est de l'huile silicone de viscosité dynamique 100 centipoise. Les deux cylindres en rotation indépendante permettent de créer un écoulement de liquide dont les caractéristiques sont :

$$
\begin{aligned}
& -1<U<1 \mathrm{~m} . \mathrm{s}^{-1} \\
& 0<G<20 \mathrm{~s}^{-1}
\end{aligned}
$$

où $G$ est le cisaillement local.

La caractérisation du champ hydrodynamique est réalisée au moyen de mesures PIV (Particle Imaging Velocimetry) pour les composantes radiales et orthoradiales. La composante axiale est mesurée par une technique LDV (Laser Doppler Velocimetry). L'écoulement strictement orthoradial au milieu des cylindres présente des recirculations importantes à proximité des bords des cylindres. Lorsque les vitesses de rotation des cylindres augmentent, des instabilités centrifuges de type rouleaux de Taylor apparaissent dans l'ensemble de l'entrefer, l'écoulement est alors tri-dimensionnel. Afin d'éviter pareil cas, la dynamique de bulles étant alors drastiquement modifiée, les mesures sont réalisées à nombre de Taylor $T a=$ $\Omega_{1}\left(r_{1}+r_{2}\right) d^{3} / 2 \nu_{\mathrm{f}}^{2}$ inférieur au nombre de Taylor critique $\left(T a_{\mathrm{C}}=1712\right.$ pour un entrefer mince $\left.d \ll r_{1}\right)$.

Dans cet écoulement, en fonction des conditions de gravité et du diamètre des bulles injectées, une large gamme de Reynolds de bulle, et de nombre de Weber peut être couverte :

$$
\begin{aligned}
& 10^{-3}<R e<10^{2} \\
& 10^{-5}<W e=\rho_{\mathrm{f}} R|U-V|^{2} / \sigma<1
\end{aligned}
$$

où $\sigma$ est la tension superficielle du liquide.

En conséquence, on s'intéressera à la prévision de la migration, donc des trajectoires des bulles dans l'entrefer, dans deux situations contrastées, l'une à nombre de Reynolds élevé avec des effets inertiels importants, et l'autre à faible nombre de Reynolds où les effets visqueux prédominent.

\section{Dynamique de bulles à grand Reynolds en condition de gravité terrestre}

La particule est soumise à plusieurs forces. Le rapport des viscosités pour une bulle d'air dans de l'huile étant faible, nous négligerons ici les effets de la force d'histoire à grand Reynolds de bulle (Magnaudet et al. [2]). Par ailleurs le mouvement de la phase fluide est supposé permanent. Les forces entrant en jeu sont donc la traînée, la force de Tchen, la force de masse ajoutée, la gravité et la portance. Les effets de déformation seront négligés dans cette partie, les nombres de Weber $W e$ étant faibles dans une grande partie des cas.

\section{La traînée}

La force de traînée s'oppose au mouvement relatif de la bulle. Elle s'exprime en fonction de la vitesse relative $(\vec{U}-\vec{V})$ et d'un coefficient de traînée $C_{\mathrm{D}}$. Mei et al. [3] proposent une expression de ce coefficient pour une bulle sphérique propre dans une large gamme de nombre de Reynolds de bulle $(1<R e<300)$ :

$$
\begin{aligned}
\vec{F}_{\mathrm{D}} & =\rho_{\mathrm{f}} C_{\mathrm{D}} \frac{\pi R^{2}}{2}|\vec{U}-\vec{V}|(\vec{U}-\vec{V}) \\
& =m_{\mathrm{f}} \frac{3}{8 R} C_{\mathrm{D}}|\vec{U}-\vec{V}|(\vec{U}-\vec{V}) \\
\text { et } \quad C_{\mathrm{D}} & =\frac{16}{R e}\left[1+\left(\frac{8}{R e}+\frac{1}{2}\left(1+\frac{3.315}{R e^{1 / 2}}\right)\right)^{-1}\right] \\
\text { avec } m_{\mathrm{f}} & =\rho_{\mathrm{f}} 4 / 3 \pi R^{3}
\end{aligned}
$$

où $m_{\mathrm{f}}$ est la masse de fluide équivalente au volume de la bulle.

La corrélation de Mei et al. tend bien vers les résultats connus : $C_{\mathrm{D}} \underset{R e \rightarrow 0}{\longrightarrow} \frac{16}{R e}$ et $C_{\mathrm{D}} \underset{R e \rightarrow \infty}{\longrightarrow} \frac{48}{R e}$ pour une bulle sphérique.

\section{La force d'inertie du liquide qui se décompose en une force de masse ajoutée et une force de Tchen}

Lorsqu'un corps se déplace, il met en mouvement autour de lui un certain volume de fluide. La force de masse ajoutée résulte de l'accélération transmise à ce volume lorsque le mouvement est instationnaire ou lorsque l'écoulement est non uniforme. Ce n'est pas seulement la 
bulle qui est accélérée, mais la bulle et la masse $C_{\mathrm{M}} m_{\mathrm{f}}$ de fluide. La force de Tchen représente la force que subirait le même volume de fluide en l'absence de bulle. Pour un écoulement de la phase fluide supposé stationnaire, elles s'écrivent :

$$
\vec{F}_{\mathrm{AM}+\mathrm{Tchen}}=m_{\mathrm{f}}\left(1+C_{M}\right) \vec{U} \cdot \nabla \vec{U}-m_{\mathrm{f}} C_{\mathrm{M}} \frac{\mathrm{d} \vec{V}}{\mathrm{~d} t}
$$

soit :

$$
\vec{F}_{\mathrm{AM}+\text { Tchen }}=-\frac{3}{2} m_{\mathrm{f}} \frac{U_{\theta}^{2}(r)}{r} \vec{e}_{r}-\frac{1}{2} m_{\mathrm{f}} \frac{\mathrm{d} \vec{V}}{\mathrm{~d} t}
$$

en supposant $C_{\mathrm{M}}=\frac{1}{2}$ pour une bulle sphérique en première approximation.

\section{La portance}

La force de portance résulte de la non uniformité de l'écoulement, elle se présente sous la forme d'une force de migration de type inertiel. Une expression classique de la force de portance est donnée, Auton [4] :

$$
\vec{F}_{\mathrm{L}}=m_{\mathrm{f}} C_{\mathrm{L}}(\vec{U}-\vec{V}) \times \vec{\Omega}=m_{\mathrm{f}} C_{\mathrm{L}}(\vec{U}-\vec{V}) \times 2 A \vec{e}_{z}
$$

À grand nombre de Reynolds de bulle, pour des particules dont la viscosité est faible devant celle du fluide, comme ici, le coefficient de portance varie faiblement avec l'intensité du cisaillement et n'est fonction que du nombre de Reynolds, Legendre \& Magnaudet [5] trouvent que pour $R e>50$ :

$$
C_{\mathrm{L}}^{R e_{P}>50}=\frac{1}{2} \frac{1+16 R e^{-1}}{1+29 R e^{-1}}
$$

avec une valeur asymptotique classique de $1 / 2$ quand le nombre de Reynolds devient grand.

Dans le cas intermédiaire où $1<R e<50$, on peut utiliser la relation suivante de Legendre \& Magnaudet [5] :

$$
C_{\mathrm{L}}=\left[\left(C_{\mathrm{L}}^{R e<1}\right)^{2}+\left(C_{\mathrm{L}}^{R e>50}\right)^{2}\right]^{1 / 2}
$$

avec, d'après Legendre \& Magnaudet [6] :

$$
C_{\mathrm{L}}^{R e<1}=\frac{6}{\pi^{2}} \frac{2.255}{\left(1+0.2 \frac{U^{2}}{G \nu_{\mathrm{f}}}\right)^{3 / 2}}\left(\frac{\nu_{\mathrm{f}}}{G R^{2}}\right)^{1 / 2}
$$

\section{La flottabilité}

$$
\vec{F}_{\mathrm{G}}=\left(m_{\mathrm{p}}-m_{\mathrm{f}}\right) \vec{g}
$$

où $m_{\mathrm{p}}$ est la masse de la bulle.

La force totale qui s'exerce sur la particule s'écrit donc de façon générale :

$$
\begin{aligned}
\vec{F}= & \vec{F}_{\mathrm{D}}+\vec{F}_{\mathrm{L}}+\vec{F}_{\mathrm{AM}+\text { Tchen }}+\vec{F}_{\mathrm{G}} \\
\vec{F}= & m_{\mathrm{f}} \frac{3}{8 R} C_{\mathrm{D}}|\vec{U}-\vec{V}|(\vec{U}-\vec{V}) \\
& +m_{\mathrm{f}} C_{\mathrm{L}}(\vec{U}-\vec{V}) \times 2 A \vec{e}_{z}-\frac{3}{2} m_{\mathrm{f}} \frac{U^{2}}{r} \vec{e}_{r} \\
& -\frac{1}{2} m_{\mathrm{f}} \vec{\Gamma}+\left(m_{\mathrm{p}}-m_{\mathrm{f}}\right) \vec{g}
\end{aligned}
$$

Contrairement à l'écoulement de la phase fluide, le mouvement de la bulle est accéléré et $\vec{\Gamma}=\mathrm{d} \vec{V} / \mathrm{d} t$ est l'accélération de la bulle. Le bilan dynamique sur la bulle s'écrit :

$$
m_{\mathrm{p}} \vec{\Gamma}=\vec{F}
$$

avec l'hypothèse d'une faible densité de la phase dispersée par rapport à celle de la phase fluide :

$$
\rho_{\mathrm{p}} \ll \rho_{\mathrm{f}} \quad \text { soit } m_{\mathrm{p}} \ll m_{\mathrm{f}} \text { et } m_{\mathrm{p}} \ll C_{\mathrm{M}} m_{\mathrm{f}}
$$

On a finalement le bilan suivant :

$$
\begin{aligned}
\vec{\Gamma}=-3 \frac{U_{\theta}^{2}(r)}{r} \vec{e}_{r}+2 C_{\mathrm{L}} & \left(U_{\theta}(r) \vec{e}_{\theta}-\vec{V}\right) \times 2 A \vec{e}_{z} \\
+ & \frac{3}{4} C_{\mathrm{D}} \frac{\left(U_{\theta}(r) \vec{e}_{\theta}-\vec{V}\right)}{\tau}-2 \vec{g}
\end{aligned}
$$

avec $\tau=R /|\vec{U}-\vec{V}|$ temps caractéristique des phénomènes d'advection.

Soit, projeté dans le repère $(r, \theta, z)$ :

$$
\begin{aligned}
\Gamma_{r} & =-\frac{3}{4 \tau} C_{\mathrm{D}} V_{r}+4 A C_{\mathrm{L}}\left(U_{\theta}-V_{\theta}\right)-3 \frac{U^{2}}{r}-2 g_{r} \\
\Gamma_{\theta} & =\frac{3}{4 \tau} C_{\mathrm{D}}\left(U_{\theta}-V_{\theta}\right)+4 A C_{\mathrm{L}} V_{r}-2 g_{\theta} \\
\Gamma_{z} & =-\frac{3}{4 \tau} C_{\mathrm{D}} V_{z}-2 g_{z}
\end{aligned}
$$

En exprimant les accélérations dans ce repère les équations précédentes deviennent :

$$
\begin{aligned}
\ddot{r}= & r \dot{\theta}^{2}-\frac{3 \dot{r}}{4 \tau} C_{\mathrm{D}}+4 A C_{\mathrm{L}}(A r+B / r-r \dot{\theta}) \\
& -3 \frac{(A r+B / r)^{2}}{r}-2 g_{r} \\
r \ddot{\theta}= & -2 \dot{r} \dot{\theta}+\frac{3}{4 \tau} C_{\mathrm{D}}(A r+B / r-r \dot{\theta})+4 A C_{\mathrm{L}} \dot{r}-2 g_{\theta} \\
\ddot{z}= & -\frac{3}{4 \tau} C_{\mathrm{D}} \dot{z}-2 g_{z}
\end{aligned}
$$

Ce problème n'a pas de solution analytique simple, l'étude de la trajectoire de la particule injectée se fera donc de manière numérique.

La spécificité de l'écoulement permet de trouver un point d'équilibre où la bulle est immobile dans le repère cylindrique $\left(\vec{e}_{r}, \vec{e}_{\theta}, \vec{e}_{z}\right)$. Dans ce cas toutes les vitesses et les accélérations étant nulles, les cylindres étant supposés parfaitement horizontaux, on obtient le système suivant :

$$
\begin{aligned}
& 4 A C_{\mathrm{L}}(A r+B / r)-3 \frac{(A r+B / r)^{2}}{r}-2 g_{r}=0 \\
& \frac{3}{4 \tau} C_{\mathrm{D}}(A r+B / r)-2 g_{\theta}=0 \\
& g_{z}=0
\end{aligned}
$$

Ainsi, reprenant l'idée de Naciri [7] dans le cas d'un écoulement de rotation solide, à partir des mesures de la position de la bulle, et connaissant les vitesses de rotation des deux cylindres, il est possible de 
Tableau 2. Récapitulatif des forces à grand Reynolds de bulle.

\begin{tabular}{lc}
\hline Force de traînée & $\vec{F}_{\mathrm{D}}=m_{\mathrm{f}} \frac{3}{8 R} C_{\mathrm{D}}|\vec{U}(r)-\vec{V}|(\vec{U}(r)-\vec{V})$ \\
\hline Force d'Archimède & $\vec{F}_{\mathrm{G}}=-m_{\mathrm{f}} \vec{g}$ \\
\hline Force de portance $\quad \vec{F}_{\mathrm{L}}=m_{\mathrm{f}} C_{\mathrm{L}}(\vec{U}(r)-\vec{V}) \times 2 A \vec{e}_{z}$ \\
\hline Force inertielle & $\vec{F}_{\mathrm{AM}+\text { Tchen }}=-\frac{3}{2} m_{\mathrm{f}} \frac{U^{2}(r)}{r} \vec{e}_{r}-\frac{1}{2} m_{\mathrm{f}} \frac{\mathrm{d} \vec{V}}{\mathrm{~d} t}$ \\
\hline
\end{tabular}

déterminer respectivement les coefficients de portance $C_{\mathrm{L}}$ et de traînée $C_{\mathrm{D}}$. C'est ce qui sera prochainement réalisé expérimentalement (Tab. 2).

\section{Dynamique de bulles en microgravité}

\subsection{Expression des forces}

En microgravité les vitesses de glissement radiales et orthoradiales étant très faibles, le régime est alors faiblement inertiel, le nombre de Reynolds $R e$ caractéristique de l'écoulement autour de la bulle est très faible mais non nul. En conséquence, la région perturbée par la présence de la bulle caractérisée par la longueur de Stokes $L_{\mathrm{S}}=R / R e$ est très importante (cf. Fig. 5), l'influence de la proximité des parois ne peut plus être négligée dans la solution des équations de Stokes. Magnaudet et al. [18], noté MTL par la suite, ont étudié l'effet de la proximité de la paroi sur le bilan dynamique pour une inclusion de densité et viscosité arbitraires (bulle, goutte, sphère solide). Ils ont établi les expressions de ces forces pour des distances $l$ de l'ordre de quelques rayons de bulles $(\kappa=R / l<0,5)$.

Pour une phase continue de forte viscosité, le nombre d'Ohnesorge est d'ordre $1: O h=R \sigma / \rho_{\mathrm{f}} \nu_{\mathrm{f}}^{2}=\mathrm{O}(1)$, les corrections sur la solution de Stokes dues aux effets d'inertie et de déformation sont du même ordre de grandeur (MTL). Dans ce cas, une force de migration induite par la déformation de la particule intervient dans le bilan dynamique. Cette force a été identifiée par Chan \& Leal [9] notamment pour des écoulements tels que $O h=o(1)$ où elle est prédominante.

Par ailleurs, bien que l'écoulement de la phase continue soit supposé stationnaire, des effets instationnaires de Masse Ajoutée et d'Histoire doivent être pris en compte à l'ordre $\mathrm{O}(\kappa)$ dans le bilan comme l'a montré Magnaudet [8].

Enfin, l'écoulement étant confiné entre deux cylindres, l'effet de la courbure de l'écoulement sera pris en compte dans le terme de Tchen et le terme de Masse Ajoutée ainsi que dans une correction à la force de migration induite par la déformation de la particule (Chan \& Leal [9]).

Dans ces conditions, les différentes forces agissant sur la bulle à proximité de la paroi sont (i) la force de traînée incluant une (ii) correction de Fàxen, (iii) la force de migration due aux effets inertiels, (iv) la force de migration due à la déformation de la bulle, $(v)$ les forces de Tchen et (vi) de masse ajoutée incluant des corrections de type force d'histoire dues à l'accélération temporelle (Magnaudet [8]).

Partant du bilan général établi par Magnaudet [8], et reprenant les calculs de Juaneda \& Colin [10], le cas d'une bulle en microgravité dans un fluide très visqueux à proximité d'une paroi va être spécifiquement étudié.

(i) L'expression de la force de traînée de Stokes quasistationnaire $\vec{F}_{\mathrm{DS}}$ établie pour une bulle par Magnaudet s'écrit de façon adimensionnelle pour un développement à l'ordre 3 en $\kappa$ :

$$
\begin{aligned}
\vec{F}_{\mathrm{DS}}= & -4 \pi \mu_{\mathrm{f}} R V_{\mathrm{C}}\left\{\left(1+\frac{3}{8} \kappa+\frac{9}{64} \kappa^{2}+\frac{27}{512} \kappa^{3}\right) \frac{\vec{V}_{\mathrm{S}}^{\theta}}{V_{\mathrm{C}}}\right. \\
& \left.+\left(1+\frac{3}{4} \kappa+\frac{9}{16} \kappa^{2}+\frac{27}{64} \kappa^{3}\right) \frac{\vec{V}_{\mathrm{S}}^{r}}{V_{\mathrm{C}}}\right\}+\mathrm{O}\left(\kappa^{4}\right)
\end{aligned}
$$

expression qui dégénère bien vers l'expression connue pour un écoulement en milieu infini loin de la paroi $(\kappa \rightarrow 0)$. Le facteur $\mu_{\mathrm{f}} R V_{\mathrm{C}}$ est dimensionnant. Les vitesses $\vec{V}_{\mathrm{S}}^{\theta}$ et $\vec{V}_{\mathrm{S}}^{r}$ sont les vitesses de glissement orthoradiale et radiale, et $V_{\mathrm{C}}$ est une échelle de vitesse caractéristique du glissement de la bulle.

(ii) En raison de l'interaction du gradient de vitesse avec la paroi, une correction de type Fàxen $\vec{F}_{\mathrm{F}}$ (MTL) doit être prise en compte :

$$
\vec{F}_{\mathrm{F}}=-\frac{\pi}{2} \mu_{\mathrm{f}} R V_{\mathrm{C}} \alpha\left(1+\frac{3}{8} \kappa\right) \kappa^{2} \vec{e}_{\theta}+\mathrm{O}\left(\kappa^{4}\right)
$$

où $\alpha=R G / V_{\mathrm{C}}$ est ici le cisaillement adimensionnel, responsable de cette force, et $G$ le cisaillement local. Une conséquence de l'existence de cette force est que la bulle va moins vite que la phase fluide dans son mouvement orthoradial.

(iii) L'expression de la force de migration inertielle due au cisaillement $\left(\vec{F}_{\mathrm{L}}\right)_{\mathrm{Sh}}$, (MTL), n'est valable que pour des faibles valeurs de $l^{*}=l / L_{\mathrm{G}}$ où $L_{\mathrm{G}}=\left(\nu_{\mathrm{f}} / G\right)^{1 / 2}$ est la longueur de Saffman caractéristique de la région sensible aux perturbations inertielles induites par la bulle. Or dans notre étude, la situation est intermédiaire entre le cas de la bulle en proche paroi de Magnaudet $(\kappa<0.5)$ et le cas asymptotique de la bulle en milieu infini de Saffman [11] où $l^{*}$ est grand. Ce type de cas intermédiaire a été étudié pour une sphère solide par McLaughlin [12]. Il a raccordé les deux comportements lorsque la particule suit les lignes de courant et est soumise à une force de portance, orthogonale à celles-ci, dans le sens du gradient de cisaillement (Fig. 3a).

Ce raccordement se fait au moyen d'un coefficient $J\left(l^{*}, \varepsilon\right)$ calculé numériquement et tabulé. Ici, le rapport $\varepsilon=\left(G \nu_{\mathrm{f}}\right)^{1 / 2} / V_{\mathrm{C}}$ des longueurs de Stokes $L_{\mathrm{S}}=$ $R / R e=\nu_{\mathrm{f}} / V_{\mathrm{C}}$ et de Saffman $L_{\mathrm{G}}$ tend vers l'infini, 


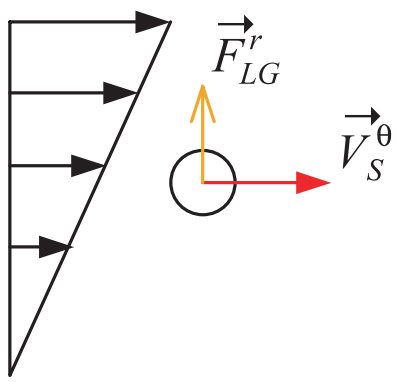

(a)

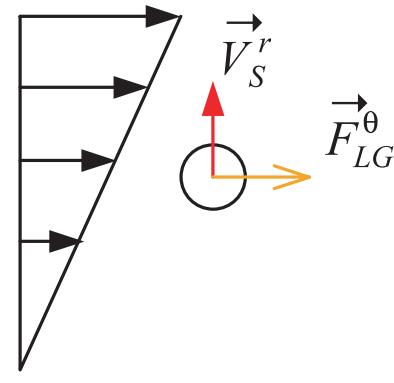

(b)
Fig. 3. Forces de migration inertielles radiales (a) et orthoradiales (b).

de sorte que la force de migration inertielle équivalente s'écrit pour une sphère solide (McLaughlin [12]) :

$$
\left\|\vec{F}_{\mathrm{LG}}\right\|=\frac{9}{\pi} \mu_{\mathrm{f}} R^{2} V_{\mathrm{S}}\left(G / \nu_{\mathrm{f}}\right)^{1 / 2} J\left(l^{*}, \varepsilon\right)
$$

On reconnaît alors en utilisant l'équation (35) de McLaughlin [12] $J=\frac{\pi^{2}}{16}\left(\frac{1}{\varepsilon}+\frac{11}{6} l^{*}\right)$, provenant de la théorie de Cox \& Hsu [17], que le terme prédominant en $l^{*}=R \kappa^{-1} / L_{\mathrm{G}}$ s'accorde avec celui en $\kappa^{-1}$ de l'équation (47b) de MTL écrit pour une sphère solide et multiplié par le Reynolds $R V_{\mathrm{C}} / \nu_{\mathrm{f}}$ caractérisant la correction de type inertielle :

$$
\begin{array}{r}
\frac{\vec{F}_{\mathrm{LG}}}{\mu_{\mathrm{f}} R V_{\mathrm{C}}}=\frac{9}{16} \pi R e\left[-\frac{11}{6} \alpha \frac{V_{\mathrm{S}}^{\theta}}{V_{\mathrm{C}}}\left(\kappa^{-1}+0,839+0,945 \kappa\right)\right. \\
\left.+\frac{55}{54} \alpha^{2}(1+0,5625 \kappa)+\left(\frac{V_{\mathrm{S}}^{\theta}}{V_{\mathrm{C}}}\right)^{2}(1+0,1875 \kappa)\right] \vec{e}_{r} \\
+\mathrm{O}\left(\kappa^{2}\right)
\end{array}
$$

Les autres termes de la force de migration inertielle de MTL utilisés ici sont à des ordres inférieurs en $\mathrm{O}\left(\kappa^{0}\right)$. On retrouve cependant que le terme en $1 / \varepsilon$ de McLaughlin s'accorde avec celui d'ordre $\mathrm{O}\left(\kappa^{0}\right)$ en $\left(V_{\mathrm{S}} / V_{\mathrm{C}}\right)^{2}$ de MTL. C'est donc ce terme $J$ qu'il faut étendre afin de raccorder les cas en proche paroi et asymptotiques de type Saffman.

De plus, on supposera que le raccordement des comportements linéarisés et asymptotiques se fait de la même façon pour le cas d'une vitesse de glissement suivant le gradient de vitesse et entraînant une force de portance dans la direction des lignes de courant de l'écoulement (Fig. 3b).

On peut donc à partir de la partie linéarisée exprimée par MTL et de la partie asymptotique, exprimée par Harper et Chang [13] pour une sphère solide, réaliser le même raccordement pour la zone intermédiaire. On obtient alors une loi de raccordement de type logarithmique obtenue pour les deux cas dans la limite $l^{*}<10$ et $\varepsilon \rightarrow \infty$ pour une sphère solide :

$$
J^{\theta}\left(l^{*}, \infty\right)=0,99 \ln \left(l^{*}\right)+1,7889
$$

et

$$
J^{r}\left(l^{*}, \infty\right)=0,5312 \ln \left(l^{*}\right)+1,0385
$$

Ces deux raccordements comparés ci-dessous aux résultas de MTL, Cox \& Hsu, et McLaughlin ne sont valables que dans le domaine $\kappa<0,5 \Rightarrow l>$ $2 R \Rightarrow l^{*}>2 R \sqrt{G / \nu}$. Les forces tracées sur la figure 4 selon les différentes expressions établies ou calculs réalisés par les différents auteurs ne contiennent que les termes divergents d'ordre $\mathrm{O}(l)$. Les résultats de MTL et Cox \& Hsu sont semblables et utilisables uniquement en proche paroi, ceux de Saffman et de Harper \& Chang sont des limites asymptotiques lorsque $l$ tend vers $+\infty$, enfin les résultats de McLaughlin proviennent d'un calcul numérique de $J\left(l^{*}, \varepsilon=\infty\right)$, pour $0,1<l^{*}<5$. Ces derniers sont valables à la fois en proche paroi et dans un écoulement de type Saffman puisque $J_{\text {McLaughlin }}\left(l^{*}, \infty\right) \underset{l^{*} \rightarrow \infty}{\longrightarrow} J_{\text {Saffman }}=2,255$.

Les résultats présenté ci-dessus ont été établis pour des valeurs données :

$$
\begin{gathered}
G=3,5 \mathrm{~s}^{-1}, \nu_{\mathrm{f}}=10^{-4} \mathrm{~m}^{2} \cdot \mathrm{s}^{-1}, \rho_{\mathrm{f}}=965 \mathrm{~kg} \cdot \mathrm{m}^{-3}, \\
V_{\mathrm{S}}^{r}=V_{\mathrm{S}}^{\theta}=1 \times 10^{-4} \mathrm{~m} \cdot \mathrm{s}^{-1}, R=10^{-3} \mathrm{~m}, \\
2 \times 10^{-3}<l<4 \times 10^{-2} \mathrm{~m}
\end{gathered}
$$

On constate que le raccordement logarithmique proposé ici suit les comportements linéaires et asymptotiques de façon satisfaisante dans les deux cas étudiés et dans la gamme de valeurs de $l^{*}$ proposée.

Par ailleurs, Legendre et Magnaudet [14] ont montré qu'il était possible de transposer les forces de portance et de traînée obtenues pour une sphère solide au cas d'une bulle en multipliant les expressions obtenues par $4 / 9 R_{\mu}^{2}$ (et non pas $R_{\mu}^{2}$ comme rapporté dans MTL et Magnaudet [8]) avec :

$$
R_{\mu}=\frac{2+3 \lambda}{2(1+\lambda)}
$$

et $\lambda=\frac{\mu}{\mu_{\mathrm{f}}} \rightarrow 0$ pour des bulles dans de l'huile.

Magnaudet [8] sépare pour une bulle la force de portance en deux contributions dont les origines physiques sont le glissement et le cisaillement (Éq. (17)). Conservant la même séparation, la force de migration reportée dans le tableau 1 est donc écrite comme une correction de nature inertielle comprenant deux contributions dues à la vitesse de glissement $\vec{F}_{\mathrm{LS}}$ :

$$
\vec{F}_{\mathrm{LS}}=\frac{\pi}{4} \rho V_{\mathrm{C}}^{2} R^{2}\left(\frac{V_{\mathrm{S}}^{\theta}}{V_{\mathrm{C}}}\right)^{2}(1+0.125 \kappa) \vec{e}_{r}+\mathrm{O}\left(\kappa^{2}\right)
$$

et au cisaillement $\vec{F}_{\mathrm{LG}}$ :

$$
\begin{aligned}
\vec{F}_{\mathrm{LG}} & =-\frac{4}{\pi} \mu_{\mathrm{f}} R^{2} \sqrt{\frac{G}{\nu}}\left(V_{\mathrm{S}}^{r} J^{\theta}\left(l^{*}, \infty\right) \vec{e}_{\theta}+V_{\mathrm{S}}^{\theta} J^{r}\left(l^{*}, \infty\right) \vec{e}_{r}\right) \\
+ & \frac{\pi}{8} \rho_{\mathrm{f}} G R^{3}\left(5 * \frac{121}{120} V_{\mathrm{S}}^{r} \vec{e}_{\theta}-\frac{11}{3}(1,125+1,023 \kappa) V_{\mathrm{S}}^{\theta} \vec{e}_{r}\right) \\
+ & \frac{\pi}{4} \frac{11}{18} \rho_{\mathrm{f}} G^{2} R^{4}(1+0,375 \kappa) \vec{e}_{r}+\mathrm{O}\left(\kappa^{2}\right)
\end{aligned}
$$

où $J^{\theta}$ et $J^{r}$ sont donnés par les relations (22) et (23). 

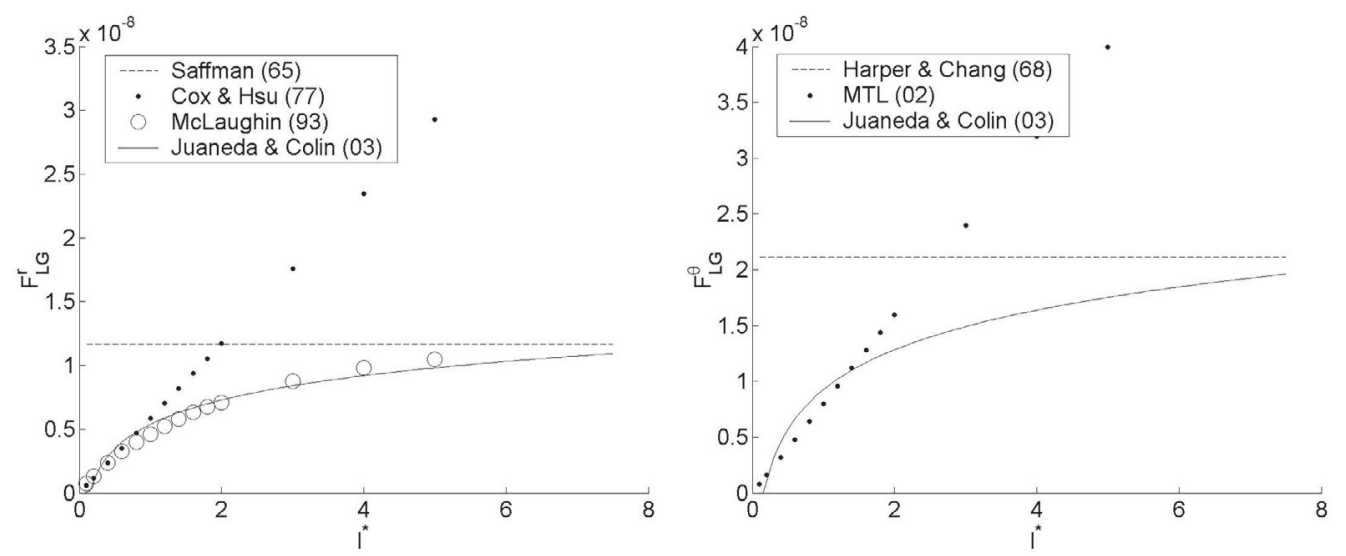

Fig. 4. Raccordement logarithmique radial et orthoradial.

Cette force est donc importante en proche paroi et son influence diminue avec $\kappa$. Elle tend à éloigner la bulle de la paroi.

(iv) En raison de la valeur du nombre d'Ohnesorge, les effets de la déformation de la bulle sur la dynamique doivent être pris en compte. MTL donnent une expression de la force de migration $\vec{F}_{\mathrm{L} \delta}$ due à la déformation pour une inclusion de viscosité et de masse volumique arbitraires. Pour $\alpha=1$, ils retrouvent ainsi, dans le cas d'une goutte en flottaison neutre dans un écoulement cisaillé linéaire, le résultat établi par Chan \& Leal [9] qui a été validé expérimentalement par Chan \& Leal [15]. Dans le cas présent, c'est le résultat obtenu par MTL, qui a apporté une correction sur l'un des facteurs, qui est utilisé. Notons que dans le cas de cette force de migration induite par la déformation, la correction calculée par MTL doit être multipliée par le nombre Capillaire $\mu_{\mathrm{f}} V_{\mathrm{C}} / \sigma$ pour obtenir la force résultante :

$$
\begin{aligned}
& \frac{\vec{F}_{\mathrm{L} \delta}}{\mu_{\mathrm{f}} R V_{\mathrm{C}}}=\frac{\mu_{\mathrm{f}} V_{\mathrm{C}}}{\sigma} \frac{\pi}{20} \\
& \times\left\{\begin{array}{l}
2\left[8 \frac{R G}{V_{\mathrm{C}}} \frac{V_{\mathrm{S}}^{\theta}}{V_{\mathrm{C}}}\left(1+\frac{3}{8} \kappa+\frac{9}{64} \kappa^{2}\right)+3\left(\frac{V_{\mathrm{S}}^{\theta}}{V_{\mathrm{C}}}\right)^{2} \kappa^{2}\right] \\
+\frac{338}{7} \frac{R^{2} G^{2}}{V_{\mathrm{C}}^{2}} \kappa^{2}
\end{array}\right\} \vec{e}_{r}
\end{aligned}
$$

Cette force strictement radiale tend comme la précédente à éloigner la bulle de la paroi, et son influence décroît avec la distance à cette paroi. Les parois seront supposées vues planes par la particule à la distance $l$ dans le repère cylindrique $\left(\vec{e}_{r}, \vec{e}_{\theta}\right)$ qui correspond au repère $\left(\vec{e}_{1}\right.$, $\vec{e}_{3}$ ) de Magnaudet.

Cependant, Chan \& Leal [9] ont montré que lorsqu'il existe un gradient du cisaillement dans l'entrefer, comme dans le cas présenté ici, la courbure du profil de vitesse donnait naissance à une vitesse de migration radiale adimensionnelle $V_{\mathrm{m}}=-8 / 14\left(B^{2} R^{4} / r^{5}\right)$ (Éq. (6.15) corrigée de Chan \& Leal [9]), qui venait se superposer à la vitesse de migration due à la déformation de la bulle. En multipliant cette vitesse de migration par le facteur $4 \pi / V_{\mathrm{C}}^{2}$, rapport des résultats de MTL (Éq. (39)) et de Chan \& Leal (Éq. (6.13), 1979), par le nombre Capillaire $\mu_{\mathrm{f}} V_{\mathrm{C}} / \sigma$, et par $\mu_{\mathrm{f}} R V_{\mathrm{C}}$ facteur dimensionnant, il est alors aisé d'exprimer la force radiale $\vec{F}_{\mathrm{C}}$ induite par la déformation due aux effets de courbure :

$$
\frac{\vec{F}_{\mathrm{C}}}{\mu_{\mathrm{f}} R V_{\mathrm{C}}}=-\frac{16}{7} \pi \frac{\mu_{\mathrm{f}} B^{2} R^{3}}{r^{5} \sigma V_{\mathrm{C}}} \vec{e}_{r}
$$

Cette force dite de « courbure $»$ sera prise en compte dans le bilan dynamique final, elle tend à attirer la bulle vers le centre des cylindres, donc vers la paroi intérieure.

(v) Comme dans le cas où $R e>50$, la force de Tchen intervient dans le bilan dynamique final à travers le terme en $\vec{U} \cdot \nabla \vec{U}$, le mouvement de la phase fluide étant supposé permanent. Cette force s'écrit donc :

$$
\vec{F}_{\mathrm{T}}=-\frac{4}{3} \pi R^{3} \rho_{\mathrm{f}} \frac{U_{\theta}^{2}(r)}{r} \vec{e}_{r}
$$

Soit, en gardant la forme générale adimensionnelle donnée par Magnaudet [8] :

$$
\frac{\vec{F}_{\mathrm{T}}}{\mu_{\mathrm{f}} R V_{\mathrm{C}}}=-\frac{4}{3} \pi \frac{R V_{\mathrm{C}}}{\nu_{\mathrm{f}}} \frac{R U_{\theta}^{2}(r)}{r V_{\mathrm{C}}^{2}} \vec{e}_{r}
$$

Cette force est ce qu'on peut qualifier d'effet « centripète » dans l'écoulement cylindrique de Couette. Elle aura toujours tendance à ramener les bulles vers le cylindre intérieur.

(vi) Dans l'écoulement étudié ici, des effets non stationnaires peuvent être pris en compte dans le bilan hydrodynamique. En effet, à la fois des effets de masse ajoutée et d'histoire peuvent apparaître pour des temps de l'ordre des variations «instantanées » de la vitesse de glissement $\vec{V}_{\mathrm{S}}$ (Magnaudet [8]). On peut ainsi reprendre pour une bulle l'expression de la force $\vec{F}_{\mathrm{U}}$ de Magnaudet :

$$
\vec{F}_{\mathrm{U}}=-\pi \operatorname{ReSt}\left\{\left[3 \kappa^{-1}+\frac{1}{4}\right] \frac{\mathrm{d} \vec{V}_{\mathrm{S} 0}^{\theta}}{\mathrm{d} t}+\left[\kappa^{-1}-\frac{1}{2}\right] \frac{\mathrm{d} \vec{V}_{\mathrm{S} 0}^{r}}{\mathrm{~d} t}\right\}
$$


Dans le cas présent, on pourra supposer que le nombre de Strouhal $S t=R / \tau V_{\mathrm{C}}=\mathrm{O}(1)$, le temps $\tau$ caractéristique des effets d'accélération étant ramené à l'échelle de la bulle. De fait, nous sommes typiquement dans le cas où $R e \ll S t \ll R e^{-1 / 2}$, c'est-à-dire que les effets non stationnaires de l'accélération et les effets quasistatiques de l'inertie sont sommables.

Dans l'expression précédente on pourra distinguer la contribution de la force de masse ajoutée, et la force d'histoire séparée en deux contributions

$$
\begin{aligned}
& \frac{\vec{F}_{\mathrm{U}}}{\mu_{\mathrm{f}} R V_{\mathrm{C}}}=\frac{\vec{F}_{\mathrm{H}}+\vec{F}_{\mathrm{AM}}}{\mu_{\mathrm{f}} R V_{\mathrm{C}}}= \\
&-\pi \frac{R V_{\mathrm{C}}}{\nu_{\mathrm{f}}} \frac{R}{V_{\mathrm{C}}^{2}}\left\{\left[3 \kappa^{-1}+\frac{1}{4}\right] \frac{\mathrm{d} \vec{V}_{\mathrm{S}}^{\theta}}{\mathrm{d} t}+\left[\kappa^{-1}-\frac{1}{2}\right] \frac{\mathrm{d} \vec{V}_{\mathrm{S}}^{r}}{\mathrm{~d} t}\right\}
\end{aligned}
$$

Dans l'expression ci-dessus la force non stationnaire a été dimensionnalisée. Notons que cette force en $\mathrm{O}\left(\kappa^{-1}\right)$ diverge lorsque l'on s'éloigne de la paroi. Elle ne peut donc être utilisée qu'en très proche paroi. Lovalenti \& Brady [16] ont obtenu une solution pour une bulle dans un écoulement sans paroi. Cependant la complexité des expressions obtenues ne permet pas un raccordement simple des deux comportements comme c'est le cas pour la force de migration inertielle.

\subsection{Calcul des forces}

Les expressions des forces ainsi établies sont valables pour une bulle à proximité d'une paroi. Afin de prendre en compte l'influence des deux cylindres, on peut remarquer que l'on a au centre de l'entrefer :

$$
\begin{aligned}
\kappa_{1}\left(=\kappa_{2}\right) \ll 1 \quad \text { et } \quad l_{1}^{*}=l_{2}^{*} \\
\text { avec } \quad \kappa_{1}=\frac{R}{\left|r-r_{1}\right|} \quad \text { et } \quad \kappa_{1}=\frac{R}{\left|r-r_{2}\right|}
\end{aligned}
$$

c'est-à-dire que les deux murs ont la même influence qui est faible puisque $\kappa \ll 1$. On fera donc dans l'entrefer l'approximation suivante :

$$
\begin{aligned}
r<\frac{r_{1}+r_{2}}{2} \Rightarrow & \vec{F}=\vec{F}\left(\kappa_{1}, l_{1}^{*}, r\right) \\
& \text { et } \quad r \geq \frac{r_{1}+r_{2}}{2} \Rightarrow \vec{F}=\vec{F}\left(\kappa_{2}, l_{2}^{*}, r\right)
\end{aligned}
$$

Le bilan total s'écrit alors pour une bulle en microgravité en régime faiblement inertiel $(\lambda \rightarrow 0, R e \ll 1)$ :

$$
\overrightarrow{0}=\vec{F}_{\mathrm{DS}}+\vec{F}_{\mathrm{F}}+\vec{F}_{\mathrm{LS}}+\vec{F}_{\mathrm{LG}}+\vec{F}_{\mathrm{L} \delta}+\vec{F}_{\mathrm{C}}+\vec{F}_{\mathrm{T}}+\vec{F}_{\mathrm{U}}
$$

Ce bilan est valable comme celui de MTL pour $\kappa<$ 0,5 et $\kappa^{-1} \ll R e^{-1}$, c'est-à-dire pour des bulles ne se trouvant pas en contact direct avec la paroi et telles que l'écoulement soit correctement décrit par les équations quasi-stationnaires de Stokes. Les différentes forces sont rappelées dans le tableau 2.

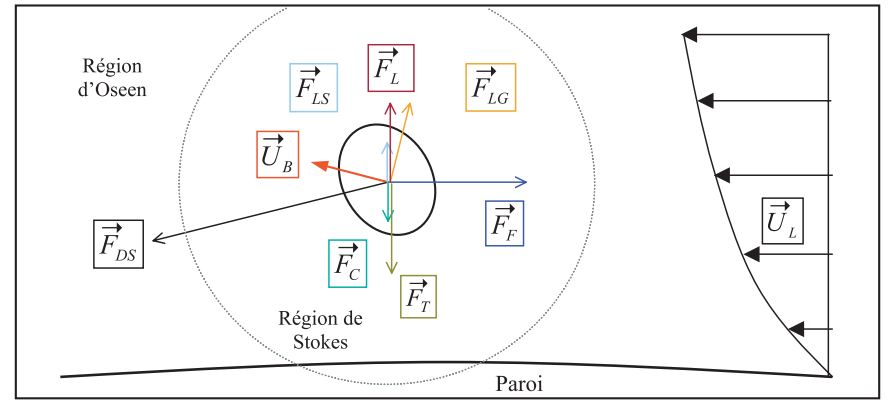

Fig. 5. Bilan dynamique de la bulle à petit Reynolds.

Il est alors possible de prédire les forces agissant sur la bulle en chaque point de l'entrefer, donc de prédire les trajectoires de ces bulles. Dans un premier temps les effets instationnaires étant négligés il est facilement possible de prédire de façon quasi-statique les vitesses de glissement ainsi que les valeurs des forces radiales et orthoradiales en tout point de l'entrefer. Le bilan dynamique (Fig. 5) est alors :

$$
\overrightarrow{0}=\vec{F}_{\mathrm{DS}}+\vec{F}_{\mathrm{F}}+\vec{F}_{\mathrm{LS}}+\vec{F}_{\mathrm{LG}}+\vec{F}_{\mathrm{L} \delta}+\vec{F}_{\mathrm{C}}+\vec{F}_{\mathrm{T}}
$$

À partir de ce bilan (Tab. 3), ces forces ont été calculées numériquement dans le cas particulier des expériences qui seront prochainement réalisées avec des bulles d'air dans de l'huile silicone. Les paramètres choisis sont :

$$
\begin{aligned}
& r_{1}=6 \mathrm{~cm}, r_{2}=10 \mathrm{~cm}, \Omega_{1}=2 \mathrm{~s}^{-1}, \Omega_{2}=-2 \mathrm{~s}^{-1}, \\
& R=1 \mathrm{~mm}, \rho_{\mathrm{f}}=965 \mathrm{~kg} \cdot \mathrm{m}^{-3}, \rho=1,225 \mathrm{~kg} \cdot \mathrm{m}^{-3}, \\
& \nu_{\mathrm{f}}=1 \times 10^{-4} \mathrm{~m}^{2} . \mathrm{s}^{-1}, \nu=1,5 \times 10^{-5} \mathrm{~m}^{2} . \mathrm{s}^{-1}
\end{aligned}
$$

Ce calcul nécessite, en tout point de l'entrefer, la résolution du système d'équations différentielles formé par le bilan dynamique projeté selon $r$ et $\theta$, et dont les deux inconnues sont les vitesses de glissement $\left(V_{\mathrm{S}}^{r}, V_{\mathrm{S}}^{\theta}\right)$. Dans le cas présent le système des forces adimensionnelles a été résolu en choisissant $V_{\mathrm{C}}=V_{\mathrm{S}}^{\theta}$, le glissement principal étant orthoradial. Cependant, dans le cas du calcul de forces dimensionnelles, il est notable que le paramètre $V_{\mathrm{C}}$ se factorise et n'est donc pas nécessaire. Les vitesses et les forces obtenues sont tracées en différents points de l'entrefer sur les figures 6 et 7 .

Dans ce cas la vitesse de glissement orthoradiale est plus importante à proximité des parois et est positive à proximité du cylindre intérieur alors qu'elle est négative à proximité du cylindre extérieur, ce dernier tournant en sens opposé. La vitesse de glissement radiale est quant à elle négative ou nulle dans tout l'entrefer. Elles sont respectivement d'ordre $10^{-5} \mathrm{~m} . \mathrm{s}^{-1}$ et $10^{-4} \mathrm{~m} . \mathrm{s}^{-1}$ (Fig. 6).

Sur la figure 7 on constate que orthoradialement, c'est la force de Fàxen qui s'oppose à la force de traînée notamment en très proche paroi, l'influence du cisaillement étant moins sensible. Au contraire dans la direction radiale, l'influence du cisaillement est prépondérante. La force de Tchen dirigée vers le centre des cylindres est responsable de la migration radiale de la bulle. 
Tableau 3. Forces s'exerçant sur une bulle en microgravité à proximité d'une paroi dans un écoulement de Couette cylindrique en régime faiblement inertiel.

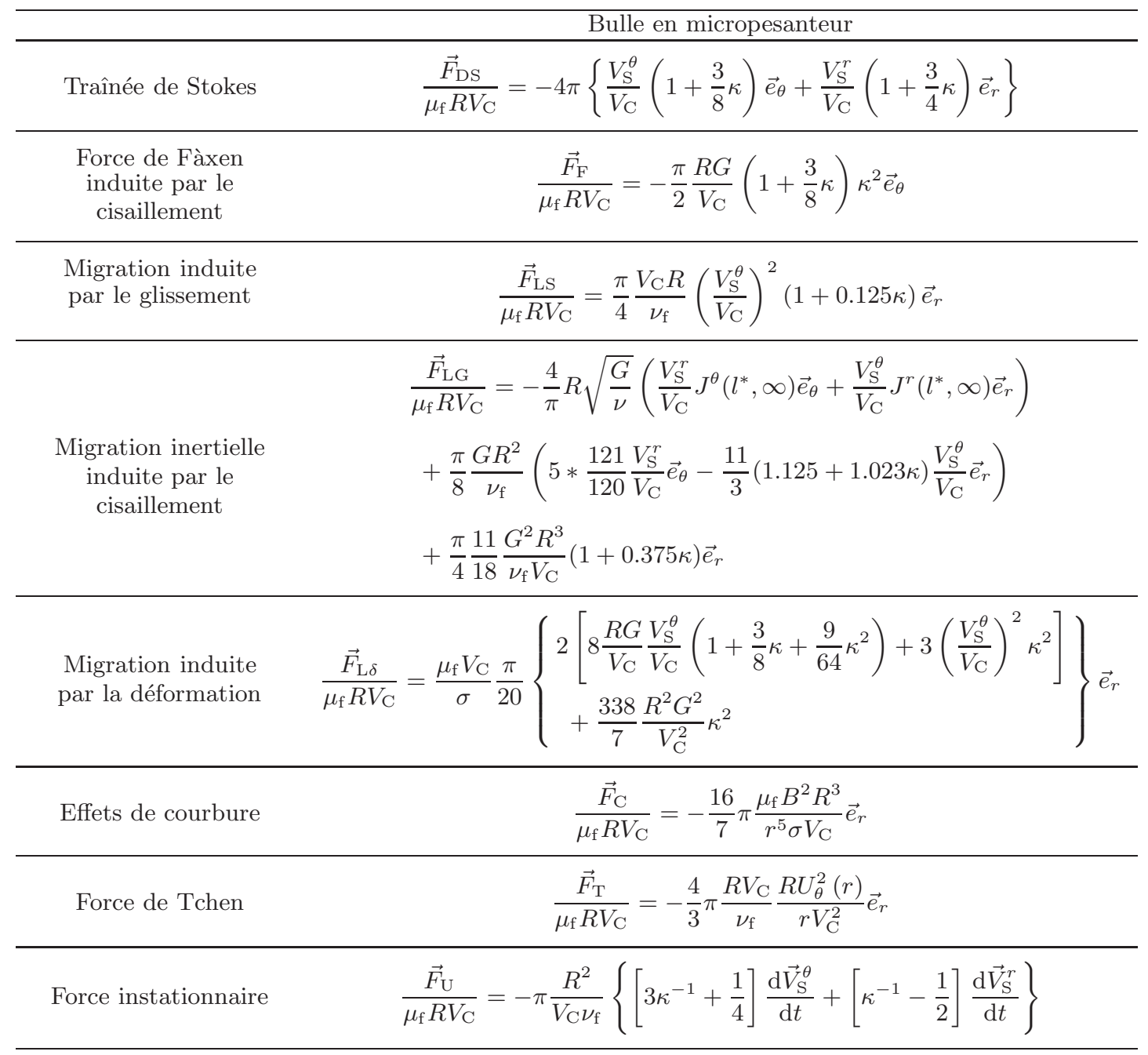

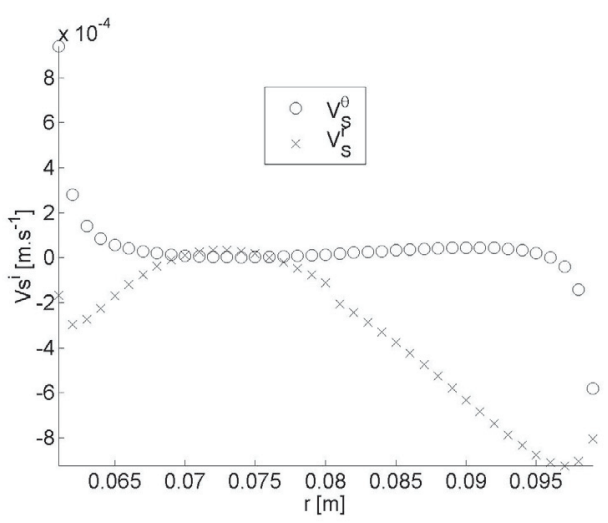

Fig. 6. Vitesses de glissement radiales et orthoradiales calculées dans l'entrefer pour l'exemple choisi.

Cette force est nulle dans l'entrefer, lorsque la vitesse $U_{\theta}(r)$ est nulle, dans ce cas les effets de migration inertiels et de déformation sont du même ordre de grandeur comme le prévoit la théorie de MTL.
Dans cette zone, l'influence des parois, et notamment la migration due au cisaillement, repousse la bulle vers le centre alors que la force de courbure tend à l'attirer systématiquement vers le cylindre intérieur, ces deux contributions étant du même ordre de grandeur. Enfin, on constate qu'en raison des faibles vitesses de glissement la portance induite par cet effet est très faible dans tout l'entrefer.

Les deux figures présentées ici (Figs. 6 et 7) ne permettent en aucun cas de tracer des trajectoires de bulles, ce qui n'est pas l'objet de cet article, elles permettent uniquement de montrer les zones d'influences de chaque force, ainsi que de comprendre physiquement leurs rôles.

\section{Conclusion}

Deux bilans dynamiques originaux s'appuyant sur des résultats obtenus dans différents cas et étendus au cas présent ont été établis afin de modéliser la migration d'une bulle dans un écoulement de Couette cylindrique 

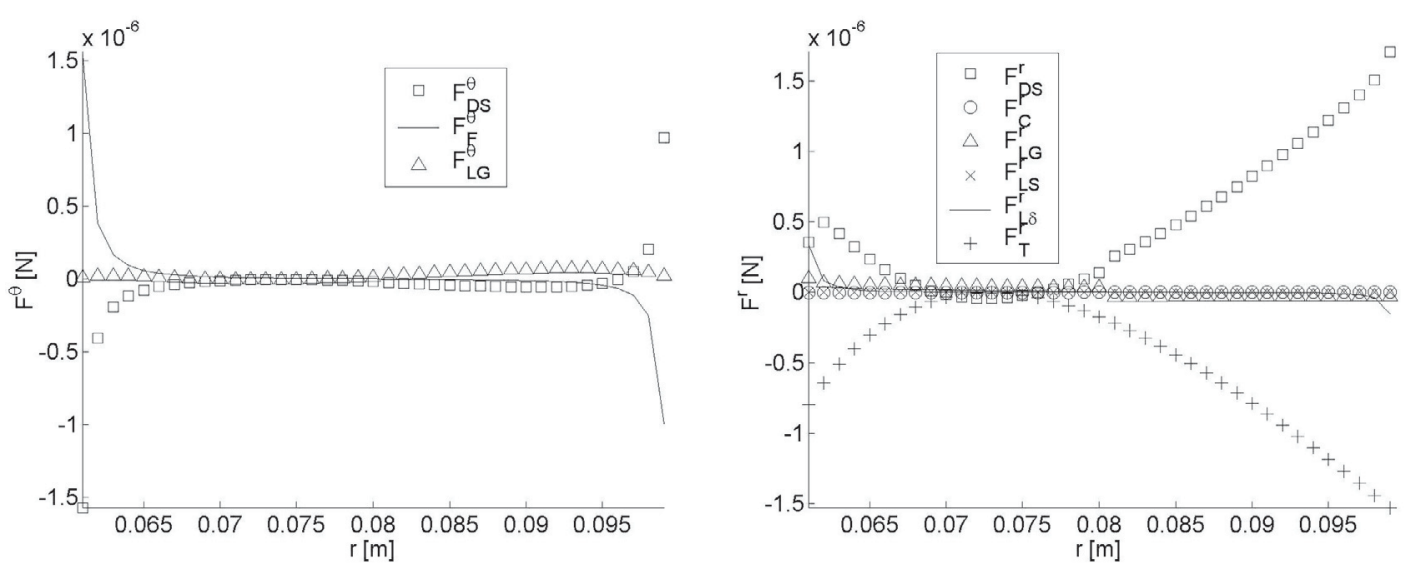

Fig. 7. Forces radiales et orthoradiales calculées dans l'entrefer pour l'exemple choisi.

en situations respectives de pesanteur terrestre et de micropesanteur. Dans le premier cas les forces mises en jeu sont classiques, mais appliquées au cas présent permettront des calculs des coefficients de traînée et de portance sur des bulles qui expérimentalement pourront être déformées. Dans le second cas, les effets du cisaillement et de la présence de la paroi sur les différentes contributions d'origines visqueuses, inertielles ou de déformation ont été pris en compte dans l'élaboration du bilan. À partir de ces expressions, les vitesses de glissement et les forces radiales et orthoradiales, hormis la force mettant en jeu les effets non stationnaires, sont obtenues dans l'entrefer en fonction des différents paramètres physiques. Afin de compléter ce bilan il faudrait prendre en compte de façon plus rigoureuse l'effet de la seconde paroi.

Des trajectoires pourront être calculées dans les deux cas après un travail numérique de discrétisations spatiales et temporelles, ainsi qu'un raccordement approprié de $\vec{F}_{\mathrm{U}}$. L'objectif suivant sera de comparer les bilans théoriques établis avec des résultats expérimentaux obtenus en laboratoire et en vols paraboliques.

Remerciements. Les auteurs tiennent à remercier le CNES et le CNRS qui supportent cette étude, ainsi que Jacques Magnaudet pour son aide précieuse sur de nombreux aspects théoriques.

\section{Références}

[1] E. Guyon, J.P. Hulin, L. Petit, Hydrodynamique Physique, CNRS Editions, EDP Sciences, 1991

[2] J. Magnaudet, M. Rivero, J. Fabre, Accelerated flows past a rigid sphere or a spherical bubble, Part 1, Steady straining flow, J. Fluid Mech. 284 (1995) 97-135

[3] R. Mei, J. Klausner, C. Lawrence, A note on the history force on a spherical bubble at finite Reynolds number, Phys. Fluids A 6 (1994) 418

[4] T.R. Auton, The lift force on a spherical body in a rotational flow, J. Fluid Mech. 183 (1987) 199-218
[5] D. Legendre, J. Magnaudet, The lift force on a spherical bubble in a viscous linear shear flow, J. Fluid Mech. 368 (1998) 81-126

[6] D. Legendre, J. Magnaudet, A note on the lift force on a spherical bubble or drop in a low-Reynolds-number shear flow, Phys. Fluids 9 (1997) 3572-3574

[7] A. Naciri, Contribution à l'étude des forces exercées par un liquide sur une bulle de gaz : portance, masse ajoutée et interactions hydrodynamiques, Thèse de doctorat, École centrale de Lyon, 1992

[8] J. Magnaudet, Small inertial effects on a spherical bubble, drop or particle moving near a wall in a time-dependent flow, J. Fluid Mech. 485 (2003) 115-142

[9] C.-H. Chan, L.G. Leal, The motion of a deformable drop in a second-order fluid, J. Fluid Mech. 92 (1979) 131-170

[10] Y. Juaneda, C. Colin, Dynamique de bulles en micropesanteur dans un écoulement de Couette en régime faiblement inertiel, Congrès Français de Mécanique, 2003

[11] P.G. Saffman, The lift force on a small sphere in a slow shear flow, J. Fluid Mech. 22 (1965) 385-400

[12] J.B. McLaughlin, The Lift on a small sphere in wallbounded linear shear flows, J. Fluid Mech. 246 (1993) 249-265

[13] E.Y. Harper, I.D. Chang, Maximum dissipation resulting from lift in a slow viscous shear flow, J. Fluid Mech. 33 (1968) 209-225

[14] D. Legendre, J. Magnaudet, The relation between the forces acting on a spherical drop or bubble and those acting on a solid sphere in the low-but-finite reynolds number regime, ITCAM 2000

[15] C.-H. Chan, L.G. Leal, An experimental study of drop migration in shear flow between concentric cylinders, Int. J. Multiphase Flow 7 (1980) 83-99

[16] P.M. Lovalentli, J.F. Brady, The force on a bubble, drop, or particle in arbitrary time-dependent motion at small Reynolds number, J. Fluid Mech. 256 (1993) 561-605

[17] R.G. Cox, S.K. Hsu, The lateral migration of solid particles in a laminar flow near a plate, Int. J. Multiphase Flow 3 (1977) 201-222

[18] J. Magnaudet, S. Takagi, D. Legendre, Drag, deformation and lateral migration of a buoyant drop moving near a wall, J. Fluid Mech. 476 (2002) 115-157 